\title{
Yang-Baxter Invariants for Line Configurations
}

\author{
R. Penne \\ Department of Mathematics and Computer Science, \\ University of Antwerp (UIA), \\ Wilrijk, Antwerp, Belgium \\ penne@hwins.uia.ac.bc
}

\begin{abstract}
We study line configurations in 3-space by means of "line diagrams," projections into a plane with an indication of over and under crossing at the vertices. If we orient such a diagram, we can associate a "contracted tensor" $T$ with it in the same spirit as is done in Knot Theory. We give conditions to make $T$ independent of the orientation, and invariant under isotopy. The Yang-Baxter equation is one such condition. Afterwards we restrict ourselves to Yang-Baxter invariants with a topological state model, and give some new invariants for line isotopy.
\end{abstract}

\section{Introduction}

There are approved methods in Knot Theory to construct link invariants from solutions of the (Quantum) Yang-Baxter Equation:

$$
R_{12} R_{13} R_{23}=R_{23} R_{13} R_{12}
$$

Here, $R$ is a linear automorphism of $V \otimes V, V$ is a vector space over a field $k$, and $R_{12}=R \otimes \mathrm{id}, \ldots$, such that (1) holds in the automorphism group of $V \otimes V \otimes V$. We refer to [5] and [9] for legible surveys on the Yang-Baxter Equation (YBE), and to [1], [8], and [14] for using it to construct link invariants. Originally, the YBE arose in Statistical Mechanics (Boltzmann weights in solvable vertex models) and in Quantum Field Theory (factorization condition for the scattering matrix).

This paper treats a new application of the YBE. We use certain solutions of it to construct isotopy invariants for line configurations in 3-space. Similarly to knots and links we can study these spatial configurations by means of diagrams, as in Fig. 1. Furthermore, the diagrams of isotopic line configurations can be connected by means of diagram moves à la Reidemeister (Fig. 2). In Section 2 we collect the facts about line configurations, diagrams, and isotopy which are necessary for our purposes. 
We always represent an element $\rho \in \operatorname{End}_{k}(V \otimes V)$ by its matrix with respect to a fixed basis. Let $\operatorname{dim}(V)=n$. A labeling of a line diagram is a choice out of $\{1, \ldots, n\}$ for every edge. It determines an entry $\rho_{c d}^{a b}$ for each vertex, taking the four incident edges in some standard order (Fig. 4). For this we need the diagram to be oriented. As a matter of fact, an orientation makes it possible to distinguish between two types of crossings (Fig. 3), giving us the opportunity to use two matrices, $\left(\rho_{c d}^{a b}\right)$ and $\left(\bar{\rho}_{c d}^{a b}\right)$. For each diagram labeling we can multiply (in the field $k$ ) the matrix entries of $\rho$ and $\bar{\rho}$ attributed to the vertices. The contracted tensor $T(\mathcal{D})$ of the given diagram $\mathcal{D}$ is obtained by doing the summation over all possible labelings. In Section 3 we deduce the properties for $\rho$ and $\bar{\rho}$ to make $T(\mathcal{D})$ independent of the chosen orientation and invariant under diagram moves. Not surprisingly, $\rho$ and $\bar{\rho}$ both have to be solutions of the YBE. The isotopy invariants for line configurations thus obtained are called Yang-Baxter invariants.

So far the Kauffman polynomial for line configurations turns out to be one of the most useful invariants for line isotopy. In Section 4 we show that the construction of Section 3 comprises an infinite number of specializations of this polynomial.

The Kauffman polynomial can be expressed by a "topological state model." A topological state of a diagram is a choice of a "resolution" for each of its crossings. A crossing can be resolved by splitting it up or by forcing an intersection. A topological state (or "resolved diagram") hence gives rise to a configuration of transversely intersecting closed curves in the projective plane. An invariant $I$ is said to have a topological state model if we can find a way to evaluate topological states such that $I$ equals the summation of these evaluations over all states. In Section 5 we derive a sufficient condition on $\rho$ such that the associated Yang-Baxter invariant has a topological state model. This condition is called the topological restriction, and it turns out to imply a significant simplification of the YBE. As a consequence we are able to characterize all Yang-Baxter invariants for $n=2$ subject to the topological restriction. Parenthetically we point out that even for the case $n=2$ it is still an open problem to find all solutions of the YBE. The ideas of Section 5 inspire in their turn state models yielding new isotopy invariants for line configurations (Section 6).

\section{Line Diagrams and Line Isotopy}

In this section we recall some definitions and results on diagrams and isotopy of line configurations, enough to reveal the motivations behind this paper. A line configuration is a configuration of $n$ nonintersecting lines in $\mathbf{R P}^{3}$. Two line configurations $\mathcal{L}_{0}$ and $\mathcal{L}_{1}$ are said to be (rigidly) isotopic if they can be connected by a continuously parametrized family $\left\{\mathcal{L}_{l} ; 0 \leq t \leq 1\right\}$ of line configurations. Viro classified line configurations up to rigid isotopy for $n \leq 5$ in [15] (see also [12]), Mazurovskii for $n \leq 6$ in [11], and Borobia and Mazurovskii for $n \leq 7$ in [2].

Two line configurations are called flexibly isotopic if they are isotopic as links in $\mathbf{R P}^{3}$. We refer to [4] for a treatment of links in $\mathbf{R P}^{3}$. All known invariants of line isotopy happen to be invariants for flexible line isotopy as well. At the time of writing it is still open whether rigid isotopy is really more restrictive than flexible isotopy for line configurations. See [3] for an extensive discussion of this matter.

A line diagram is a simple arrangement of lines in the affine plane, equipped with over 


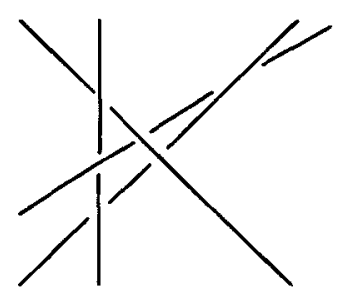

Fig. 1. A line diagram.

and under crossings, in the same spirit as knot diagrams (Fig. 1). The underlying line arrangement of a line diagram is called its universe. We consider a line diagram always up to global affine transformations of its universe. Two line diagrams whose universes can be mapped to each other by a self-homeomorphism of the affine plane, and with the same under/over crossings for corresponding vertices, are said to be combinatorially equivalent. Relaxing the geometric character of the universe, and focusing on its combinatorial type, automatically leads to the concept of a pseudoline diagram: a simple arrangement of "pseudolines" with over and under crossings.

For our purposes a pseudoline is the image of an affine line under a self-homeomorphism of $\mathbf{R}^{2}$. Furthermore, we always assume that an affine pseudoline can be "extended" to a projective pseudoline in the projective closure $\mathbf{R P}^{2}$. Pseudoline diagrams are considered up to combinatorial equivalence, akin to knot diagrams. We refer to [13] for an introduction to pseudoline diagrams. In that paper we introduced two types of moves on pseudoline diagrams: $\|$-moves and $*$-moves (Fig. 2). Observe that $\|$-moves correspond to vertices passing the "line at infinity," while $*$-moves must be compared with the third Reidemeister move. Two pseudoline diagrams are called equivalent if they can be transformed to each other by means of a sequence of $\|$-moves and $*$-moves. Two line diagrams are said to be equivalent if they are equivalent as pseudoline diagrams.
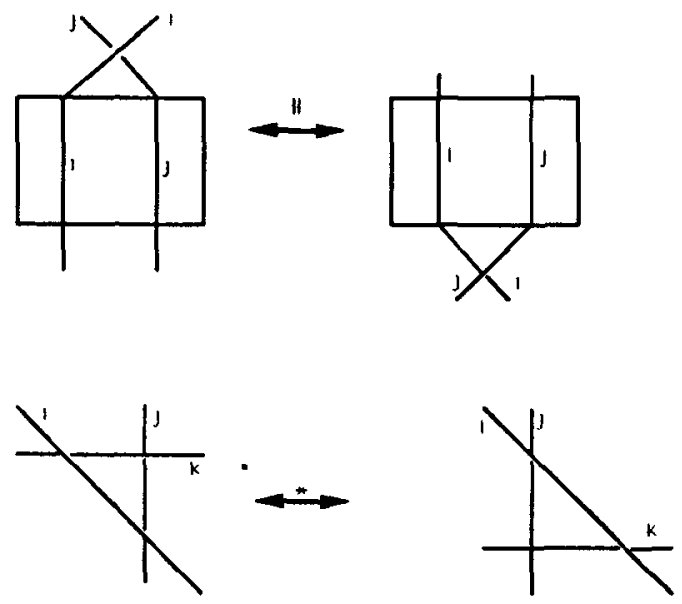

Fig. 2. Illustration of a $\|$-move and a *-move. 
Suppose we are given two line configurations $\mathcal{L}_{0}$ and $\mathcal{L}_{1}$ in $\mathbf{R P}{ }^{3}$. We can always choose the plane at infinity $\pi_{\infty}$ in $\mathbf{R} \mathbf{P}^{3}$ transverse to both configurations, i.e., $\pi_{\infty}$ does not contain any line if $\mathcal{L}_{i}(i=0,1)$. As a result, we obtain two configurations of mutually skew lines in $\mathbf{R}^{3}$, which can be projected to simple arrangements in some affine plane. Reporting the relative heights along the direction of projection for every vertex finally yields two line diagrams $\mathcal{D}_{0}$ and $\mathcal{D}_{1}$, called the planar layouts of $\mathcal{L}_{0}$ and $\mathcal{L}_{1}$, respectively. In [3] we proved that $\mathcal{D}_{0}$ and $\mathcal{D}_{1}$ are equivalent if $\mathcal{L}_{0}$ and $\mathcal{L}_{1}$ are isotopic. In the same paper geometric conditions are given which make the converse statement become true, yielding a Reidemeister-like theorem for line isotopy. This provides us with a general strategy to design invariants for line isotopy: define an entity on pseudoline diagrams which does not alter under $\|$-moves and $*$-moves.

\section{Tensor Contraction on Line Diagrams}

Let $k$ be a field, let $V$ be a finite-dimensional vector space over $k$, and let

$$
\rho: V \otimes V \rightarrow V \otimes V
$$

be a $k$-linear map. If we fix a basis $\left\{e_{1}, \ldots, e_{n}\right\}$ of $V$, then we write $\left(\rho_{c d}^{a b}\right)$ for the $\left(n^{2} \times n^{2}\right)$-matrix associated with $\rho$ :

$$
\rho\left(e_{c} \otimes e_{d}\right)=\rho_{c d}^{a b} e_{a} \otimes e_{b}
$$

Here, and everywhere else in this article, Einstein's convention of summation is used.

Suppose we are given a (pseudo)line diagram $\mathcal{D}$ with $m \geq 1$ (pseudo)lines. We construct a (contracted) tensor $T(\mathcal{D})$ by means of two elements of $\operatorname{End}_{k}(V \otimes V), \rho$ and $\bar{\rho}$. To this end, we first have to choose a direction in the affine plane, a unit vector $\vec{u}$ not perpendicular to any of the lines of $\mathcal{D}$, inducing an orientation for each of the lines of $\mathcal{D}$. This enables us to distinguish between two types of crossings (Fig. 3). If $\mathcal{H}$ is the underlying universe of the diagram $\mathcal{D}$, then we can consider its projective closure $\overline{\mathcal{H}} \subset \mathbf{R} \mathbf{P}^{2}$. By definition we say that the edges of $\mathcal{D}$ are the connected components of the complement of the $\left(\begin{array}{c}m \\ 2\end{array}\right)$ vertices in $\bigcup \overline{\mathcal{H}}$. This means that two unbounded (affine) edges on the same pseudoline of $\mathcal{H}$ are regarded as one edge, connecting two vertices. A state $\sigma$ of $\mathcal{D}$ is a map from the edges of $\mathcal{D}$ to $\{1, \ldots, n\}$, that is, $\sigma$ gives a labeling of the edges. Given such a state $\sigma$ we can assign a scalar $W(v)$ to each vertex $v$ of $\mathcal{H}$, depending on whether it is a positive or a negative crossing of $\mathcal{D}$, called a vertex weight. More precisely, if the incoming edges of $v$ are labeled by $(a, b)$ and the outgoing edges by $(c, d)$, moreover appearing in the order as given in Fig. 4 , then $W(v)=\rho_{c d}^{a b}$ or $\bar{\rho}_{c d}^{a b}$,
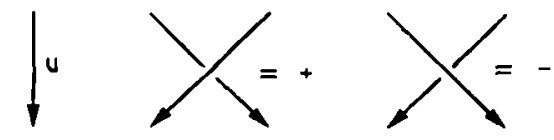

Fig. 3. A positive crossing on the left and a negative crossing on the right. 

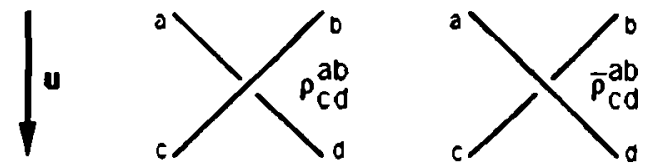

Fig. 4. Vertex weights for positive and negative crossings, using representations of $\rho$ and $\bar{\rho}$ in a fixed basis.

depending on whether $v$ is positive or negative, respectively. Further, let

$$
\langle\mathcal{D} \mid \sigma\rangle_{\bar{u}}=\prod_{\text {vertices } v} W(v)
$$

be the product ${ }^{1}$ of all vertex weights thus obtained. Then we can define

$$
T(\mathcal{D})_{\vec{u}}=\sum_{\text {states } \sigma}(\mathcal{D} \mid \sigma)_{\vec{u}}
$$

Notice that in case $\mathcal{D}$ contains only one line $(m=1), T(\mathcal{D})_{\vec{u}}=n$.

Example. For the diagram of Fig. 5, using the indicated direction $\vec{u}$, we get

$$
T(\mathcal{D})_{\vec{u}}=\rho_{d e}^{b c} \bar{\rho}_{c f}^{a d} \rho_{b a}^{f e},
$$

with summation over repeated indices.

We first detect conditions on $\rho$ and $\bar{\rho}$ such that $T(\mathcal{D})_{\vec{u}}$ becomes independent from $\vec{u}$. To this end, we consider the four essentially different positions of $\vec{u}$ for a fixed crossing with labeled edges. We refer to Fig. 6 . We conclude that the conditions

$$
\begin{aligned}
& \rho_{c d}^{a b}=\rho_{b a}^{d c}, \\
& \bar{\rho}_{c d}^{a b}=\rho_{a c}^{b d}
\end{aligned}
$$

are sufficient to have for each pair of directions $\left\{\vec{u}_{1}, \vec{u}_{2}\right\}$ that

$$
T(\mathcal{D})_{\vec{u}_{1}}=T(\mathcal{D})_{\bar{u}_{2}} .
$$

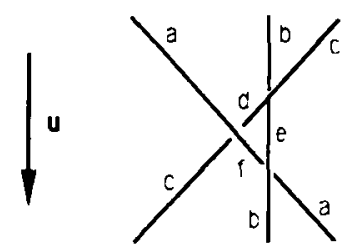

Fig. 5. A line diagram with labeled edges.

\footnotetext{
1 The empty product is defined to be 1 .
} 

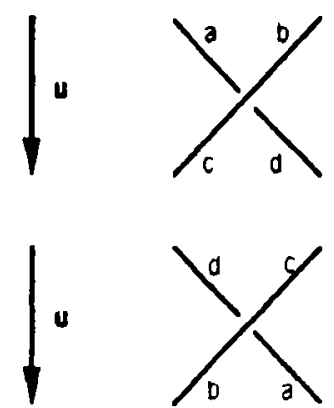
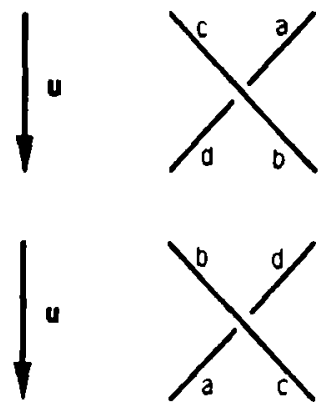

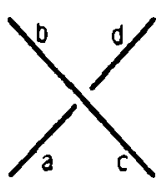

Fig. 6. Four different views of the same crossing with labeled edges.

From now on we assume that $\rho$ and $\bar{\rho}$ satisfy (2) and (3) (with respect to the chosen basis), leading to a scalar $T(\mathcal{D})$ independent from $\vec{u}$. If not mentioned otherwise, it is assumed in the figures that $\vec{u}$ points from "top to bottom." Observe that (2) and (3) together imply that

$$
\bar{\rho}_{c d}^{a b}=\bar{\rho}_{b a}^{d c} .
$$

Next, we want $T(\mathcal{D})$ to be invariant under diagram moves. We first perform a $\|$ move, which can be assumed to be as in Fig. 7 by choosing an appropriate orientation, up to changing the sign of the crossing. Because $\|$-moves do not affect the projective closure of the universe we can compare corresponding states before and after. Fix a state $\sigma$. Since vertices and vertex weights in the box have not been altered (Fig. 7), neither has their product $B_{\sigma}$. The requirement of invariance of $\left.\langle\mathcal{D}| \sigma\right)$ under $\|$-moves comes down to

$$
\rho_{c d}^{a b} B_{\sigma}=B_{\sigma} \rho_{d c}^{b a} \quad \text { or } \quad \bar{\rho}_{c d}^{a b} B_{\sigma}=B_{\sigma} \bar{\rho}_{d c}^{b a},
$$

depending on the sign of the involved crossing. We derive the following conditions:

$$
\begin{aligned}
& \rho_{c d}^{a b}=\rho_{d c}^{b a}, \\
& \bar{\rho}_{c d}^{a b}=\bar{\rho}_{d c}^{b a} .
\end{aligned}
$$

Notice that (2) and (5) (resp. (4) and (6)) imply that $\rho$ (resp. $\bar{\rho}$ ) is represented by a
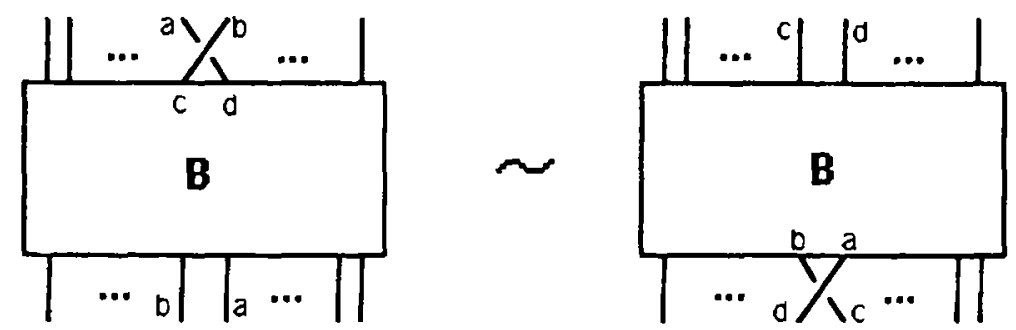

Fig. 7. The effect of $\|$-moves on $T(\mathcal{D})$. 

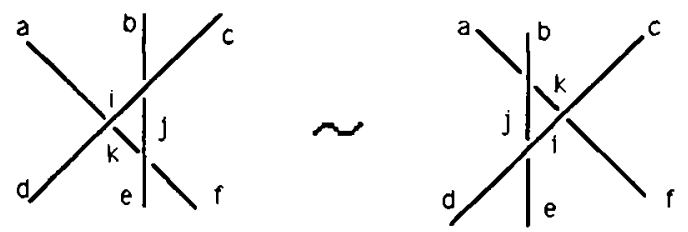

Fig. 8. The effect of $*$-moves on $T(\mathcal{D})$.

symmetric matrix with respect to the fixed basis:

$$
\begin{aligned}
& \rho_{c d}^{a b}=\rho_{a b}^{c d}, \\
& \bar{\rho}_{c d}^{a b}=\bar{\rho}_{a b}^{c d} .
\end{aligned}
$$

To establish invariance under $*$-moves, it suffices to consider only the cases of three crossings with equal signs (Fig. 8). This is due to the fact that we can freely choose the orientations. In order to compare $T(\mathcal{D})$ before and after a $*$-move, we fix equal labels for corresponding edges outside the involved triangle, and do the summation over all possible labels for the triangle edges. This means that $\rho$ and $\bar{\rho}$ have to obey the following $n^{6}$ YBEs (Fig. 8):

$$
\begin{aligned}
& \rho_{i j}^{b c} \rho_{d k}^{a i} \rho_{e f}^{k j}=\rho_{j k}^{a b} \rho_{i f}^{k c} \rho_{d e}^{j i}, \\
& \bar{\rho}_{i j}^{b c} \bar{\rho}_{d k}^{a i} \rho_{e f}^{k j}=\bar{\rho}_{j k}^{a b} \bar{\rho}_{i f}^{k c} \bar{\rho}_{d e}^{j i} .
\end{aligned}
$$

We conclude in

Theorem 1. If $\rho \in \operatorname{End}_{k}(V \otimes V)$ corresponds to the matrix $\left(\rho_{c d}^{a b}\right)$ with respect to some basis $\left\{e_{1}, \ldots, e_{n}\right\}$ of $V$, and if $\bar{\rho} \in \operatorname{End}_{k}(V \otimes V)$ is defined by $\bar{\rho}_{c d}^{a b}=\rho_{a c}^{b d}$, then the conditions

(1) $\rho$ and $\bar{\rho}$ satisfy the YBEs,

(2) $\left(\rho_{c d}^{a b}\right)$ and $\left(\bar{\rho}_{c d}^{a b}\right)$ are symmetric matrices,

imply that $T(\mathcal{D})$ is an invariant for diagram equivalence.

Proof. Using relations (3) and (8) we obtain

$$
\rho_{c d}^{a b}=\bar{\rho}_{d b}^{c a}=\bar{\rho}_{c a}^{d b}=\rho_{d c}^{b a},
$$

that is, condition (5). In the same way we can prove condition (6). The theorem is proven by observing that (5) and (7) together imply (2), and that (6) and (8) give (4).

\section{Remarks}

- Conditions (7) and (8) in Theorem 1 can be replaced by conditions (5) and (6).

- Under the conditions of Theorem $1, \bar{\rho}$ can be alternatively defined as

$$
\bar{\rho}_{c d}^{a b}=\rho_{b d}^{a c} .
$$


- Invariants $T(\mathcal{D})$ as introduced in Theorem 1 are called Yang-Baxter invariants for diagram equivalence (or for line isotopy).

\section{An Example: the Kaufiman Polynomial}

Take $A$ and $B$ in $k$. We define $\rho \in \operatorname{End}(V \otimes V)$ by

$$
\begin{aligned}
& \rho\left(e_{a} \otimes e_{b}\right)=A e_{a} \otimes e_{b} \quad(a \neq b), \\
& \rho\left(e_{a} \otimes e_{a}\right)=A e_{a} \otimes e_{a}+\sum_{b=1}^{n} B e_{b} \otimes e_{b},
\end{aligned}
$$

which can be expressed in matrix form as

$$
\rho_{c d}^{a \grave{b}}=A \delta_{c}^{a} \delta_{d}^{b}+B \delta^{a b} \delta_{c d}
$$

and which is apparently symmetric. Further,

$$
\bar{\rho}_{c d}^{a b}=\rho_{a c}^{b d}=B \delta_{c}^{a} \delta_{d}^{b}+A \delta^{a b} \delta_{c d},
$$

which is symmetric as well.

The $k$-endomorphism given by formula (11) is well known, since it yields Kauffman's "state model" for the Jones polynomial (see [7]). A state $\sigma$ (in the sense of Section 3) that contributes a nonzero term $\langle\mathcal{D}| \sigma)$ in $T(\mathcal{D})$, assigns labels to the four incident edges of a crossing in either one of two ways, as shown in Fig. 9, which can be represented by "splitting" the vertices in either of two ways. If we resolve thus all the crossings of the diagram $\mathcal{D}$, we come up with a collection of disjoint closed curves in $\mathbf{R P}^{2}$ where each curve has the property that it links edges with the same label. We call such a configuration of closed curves a split state $\Sigma$ of $\mathcal{D}$, in order to distinguish it from the states of Section 3 . Observe that if $\sigma$ selects the same label for all four edges of some crossing $(a=b)$, it corresponds to several split states. Finally, the weight of a split state $\Sigma$ is defined by evaluating the way it splits the crossings, distinguishing $A$-splits from $B$-splits (Fig. 10):

$$
\langle\mathcal{D} \mid \Sigma\rangle=n^{|\Sigma|} A^{a(\Sigma)} B^{b(\Sigma)},
$$

where $|\Sigma|$ is the number of loops in $\Sigma, n=\operatorname{dim}_{k} V, a(\Sigma)$ is the number of $A$-splits in $\Sigma$, and $b(\Sigma)$ is the number of $B$-splits in $\Sigma$.

It is not hard to see that

$$
T(\mathcal{D})=\sum_{\text {split states }}\langle\mathcal{D} \mid \Sigma\rangle
$$
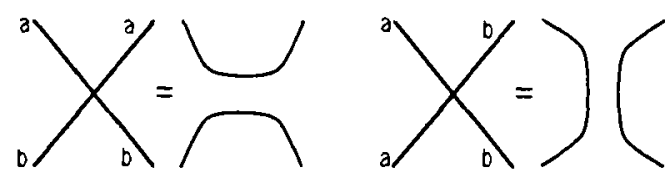

Fig. 9. The only two nonzero vertex weights, and the associated vertex splits. 


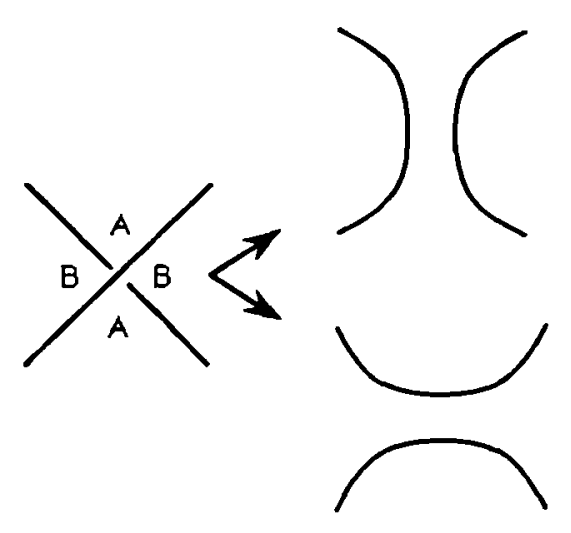

Fig. 10. The $A$-split and the $B$-split of a crossing.

To be an invariant for line isotopy, $\rho$ and $\bar{\rho}$ should satisfy (9) and (10), respectively. It is well known (e.g., $[8])$ that $\rho$ and $\bar{\rho}$ satisfy the YBE if and only if

$$
\begin{aligned}
& A\left(A^{2}+n A B+B^{2}\right)=0, \\
& B\left(A^{2}+n A B+B^{2}\right)=0,
\end{aligned}
$$

respectively. So, if we take $A$ and $B$ in $k$ such that $A^{2}+n A B+B^{2}=0$, and if we define $\rho$ and $\bar{\rho}$ by (11) and (12), then the abstracted tensor $T(\mathcal{D})$ is an invariant for line isotopy. It is convenient to regard $A$ and $B$ as variables, and

$$
T(\mathcal{D}) \in \mathbf{Z}[A, B] /\left(A^{2}+n A B+B^{2}\right)
$$

Remark. In previous articles on line isotopy (e.g., [3], [10], [13]) it has already been observed that Kauffman's model for the Jones polynomial can be defined for line diagrams by considering the split states in the projective plane. More precisely, the Kauffman polynomial for line configurations has been defined as

$$
K(\mathcal{D})=\sum_{\text {split states }} A^{a(\Sigma)} B^{b(\Sigma)} d^{|\Sigma|}
$$

with $A^{2}+d A B+B^{2}=0$. As a matter of fact, it is a special case of the construction of the Jones polynomial for links in $\mathbf{R P}^{3}$, as was done in [4] (where one must put $B=A^{-1}$ ). Mazurovskii proved that $K(\mathcal{D})$ distinguishes the isotopy types of configurations up to six lines [10].

The previous example provides a Yang-Baxter model for an infinite number of specializations for $K(\mathcal{D})(d=n)$. It would be interesting to rediscover the unspecialized $K(\mathcal{D})$ as a Yang-Baxter model, as it has been established for link diagrams [8]. 


\section{The Topological Restriction}

Section 4 shows an example of a Yang-Baxter invariant which is representable by a "topological model." In general, a topological state of a given (pseudo)line diagram is a choice at each crossing of how to "resolve" it. This can involve $A$-splits and $B$-splits (as in Kauffman's model), or the change of a crossing into a real intersection. Such a topological state gives rise to a configuration of closed curves in the projective plane, transversely intersecting each other. Most often, we assume that spins are involved, with labels from 1 to $n$ assigned to each of these curves (labeled topological states). Further, we let a weight, which may depend on the type of resolution and the local spin values, correspond to each resolved crossing. The global evaluation of a topological state is done by multiplying the local weights of the resolutions. An isotopy invariant for diagrams is said to have a topological model if it can be expressed as the summation of global evaluations over all (labeled) topological states. For a great variety of link invariants it is known that they can be represented by similar topological models (see [6] and [8]). ${ }^{2}$

This motivates us to impose an extra condition on the matrices $\left(\rho_{c d}^{a b}\right)$ and $\left(\bar{\rho}_{c d}^{a b}\right)$ of Theorem 1,

$$
\rho_{c d}^{a b} \neq 0 \Rightarrow\left\{\begin{array}{l}
a \neq b \Rightarrow\{a, b\}=\{c, d\} \\
a=b \Rightarrow c=d,
\end{array}\right.
$$

called the topological restriction. This means that only $\rho_{a b}^{a b}, \rho_{b b}^{a a}, \rho_{b a}^{a b}$ and $\bar{\rho}_{a b}^{a b}, \bar{\rho}_{b b}^{a a}, \bar{\rho}_{b a}^{a b}$ stand a chance of being nonzero $(a, b=1, \ldots, n)$, reducing the number of relevant edge labelings of a given diagram. If we associate $A$-splits (resp. $B$-splits) with $\rho_{a b}^{a b}$ (resp. $\bar{\rho}_{b b}^{a a}$ ), and $B$-splits (resp. $A$-splits) with $\rho_{b b}^{a a}$ (resp. $\bar{\rho}_{a b}^{a b}$ ), and intersections with $\rho_{b a}^{a b}$ and $\bar{\rho}_{b a}^{a b}$, then the contracted tensor $T(\mathcal{D})$ has a topological model. Observe that the vertex weights $\rho_{a a}^{a a}$ and $\bar{\rho}_{a a}^{a a}$ correspond to different resolutions. In order to avoid self-intersecting curves we model $\rho_{b a}^{a b}$ and $\bar{\rho}_{b a}^{a b}$ by intersections only if $a \neq b$.

For example, in the case $n=2$, the topological restriction means that $\rho$ and $\bar{\rho}$ can be represented by the following matrices:

$$
\rho=\left(\begin{array}{llll}
p & 0 & 0 & t \\
0 & q & s & 0 \\
0 & s & q & 0 \\
t & 0 & 0 & r
\end{array}\right), \quad \bar{\rho}=\left(\begin{array}{cccc}
p & 0 & 0 & q \\
0 & t & s & 0 \\
0 & s & t & 0 \\
q & 0 & 0 & r
\end{array}\right) .
$$

Notice that the equalities between the nonzero entries in ansatz (16) are implied by the symmetry of $\rho$ and $\bar{\rho}$. Equivalently, we can express $\rho$ (resp. $\bar{\rho}$ ) as an expansion of resolutions of a positive (resp. negative) crossing (Fig. 11). If each crossing is resolved we get a configuration $\Sigma$ of (not necessarily disjoint) non-self-intersecting closed curves in $\mathbf{R P}^{2}$ that carry a constant label ( 1 or 2 ). Unlike Kaufmann's model for the Jones polynomial, the vertex weights of these split states depend on the loop labels.

We now investigate to what extent the system of $n^{6}$ YBEs is simplified by conditions (7), (5), and (15). To this end we introduce some notation. With each choice

\footnotetext{
2 In Knot Theory a topological state is a configuration of closed curves in the affine plane, whose global evaluation often requires an additional factor depending on global topological properties (such as winding numbers).
} 


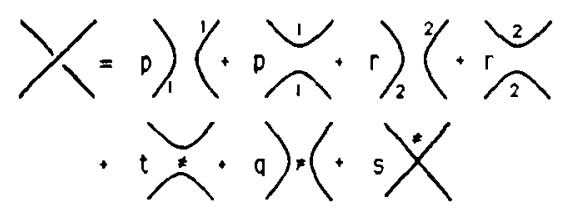

Fig. 11. Expression of $\rho$ as an expansion of a crossing into resolutions. The weight of each resolution depends on the selected "spins."

of a 6 -tuple $(a, b, c, d, e, f) \in\{1, \ldots, n\}^{6}$ an equation $Y B(a, b, c, d, e, f)$ of (9) is associated:

$$
L H S(a, b, c, d, e, f)=\operatorname{RHS}(a, b, c, d, e, f) .
$$

Further, each term of the LHS (resp. RHS) is determined by specifying the values for the summation indices $(i, j, k) \in\{1, \ldots, n\}^{3}$, denoted by $L T_{i j k}\left(\operatorname{resp} . R T_{i j k}\right)$.

Lemma 2. Let $\left(\rho_{c d}^{a b}\right)$ be a solution of the YBE subject to the topological restriction (15). Choose $(a, b, c, d, e, f) \in\{1, \ldots, n\}^{6}$. If some value $x \in\{1, \ldots, n\}$ occurs an odd number of times in $(a, \ldots, f)$, then all terms of $Y B(a, \ldots, f)$ are identically zero.

Proof. Let $T=L T_{i j k}$ or $R T_{i j k}$ be an arbitrary term in $Y B(a, \ldots, f)$. We can write $r=F_{1} F_{2} F_{3}$, each factor $F_{r}$ being an entry of the matrix $\left(\rho_{c d}^{a b}\right)$. Observe that $F_{r}$ contains exactly two parameters

$$
\left\{x\left(F_{r}\right), y\left(F_{r}\right)\right\} \subset\{a, \ldots, f\}
$$

and two summation indices

$$
\left\{I\left(F_{r}\right), J\left(F_{r}\right)\right\} \subset\{i, j, k\}
$$

Assume now that $T \neq 0$. We distinguish two cases.

Case 1. There is a factor $F_{i}$ of $T$ with $x\left(F_{i}\right)=y\left(F_{i}\right)$; without loss of generality we assume that $x\left(F_{1}\right)=y\left(F_{1}\right)$. If $T \neq 0$, condition (15) implies that $I\left(F_{1}\right)=J\left(F_{1}\right)$. Since each summation index occurs in exactly two factors of $T$, we can state that

$$
I\left(F_{2}\right)=I\left(F_{1}\right)=J\left(F_{1}\right)=J\left(F_{3}\right)
$$

Notice that $J\left(F_{2}\right)$ always equals $I\left(F_{3}\right)$. If $J\left(F_{2}\right)=I\left(F_{2}\right)$, then $I\left(F_{3}\right)=J\left(F_{3}\right)$, implying both $x\left(F_{2}\right)=y\left(F_{2}\right)$ and $x\left(F_{3}\right)=y\left(F_{3}\right)$. While if $J\left(F_{2}\right) \neq I\left(F_{2}\right)$, and hence $I\left(F_{3}\right) \neq$ $J\left(F_{3}\right)$, it follows that

$$
\left\{x\left(F_{2}\right), y\left(F_{2}\right)\right\}=\left\{I\left(F_{2}\right), J\left(F_{2}\right)\right\}=\left\{I\left(F_{3}\right), J\left(F_{3}\right)\right\}=\left\{x\left(F_{3}\right), y\left(F_{3}\right)\right\}
$$

In any case, each value in $\{1, \ldots, n\}$ is taken an even number of times by $(a, \ldots, f)$. 
Case 2. We have $x\left(F_{1}\right) \neq y\left(F_{1}\right), x\left(F_{2}\right) \neq y\left(F_{2}\right)$, and $x\left(F_{3}\right) \neq y\left(F_{3}\right)$. So, $I\left(F_{r}\right) \neq J\left(F_{r}\right)$ for each factor $F_{r}$. This implies that, for all $1 \leq r \neq s \leq 3$,

$$
\left|\left\{I\left(F_{r}\right), J\left(F_{r}\right)\right\} \cap\left\{I\left(F_{s}\right), J\left(F_{s}\right)\right\}\right|=1
$$

and

$$
\left\{I\left(F_{1}\right), J\left(F_{1}\right)\right\} \cap\left\{I\left(F_{2}\right), J\left(F_{2}\right)\right\} \cap\left\{I\left(F_{3}\right), J\left(F_{3}\right)\right\}=\varnothing .
$$

Since on the other hand $\left\{x\left(F_{r}\right), y\left(F_{r}\right)\right\}=\left\{I\left(F_{r}\right), J\left(F_{r}\right)\right\}$, we conclude that each element of $\{1, \ldots, n\}$ occurs an even number of times in $\left(x\left(F_{1}\right), y\left(F_{1}\right), x\left(F_{2}\right), y\left(F_{2}\right), x\left(F_{3}\right)\right.$, $y\left(F_{3}\right)$ ) (more precisely, 0 or 2 ).

Lemma 3. Let $\left(\rho_{c d}^{a b}\right)$ be a solution of the YBE subject to the topological restriction (15). If $a=b=\cdots=f$, then $\operatorname{LHS}(a, \ldots, f)$ is identical to $R H S(a, \ldots, f)$.

Proof. By condition (15) it follows that $i=j=k$ for each nonzero term of $Y B(a, \ldots, f)$. Consequently,

$$
L T_{i j k} \equiv R T_{i j k} .
$$

So, each nonzero term of the LHS cancels with the corresponding term of the RHS.

Each choice $(a, \ldots, f) \in\{1, \ldots, n\}^{6}$ partitions the parameter set $\{a, \ldots, f\}$ in classes of equal values. From the two previous lemmas we learn that if $Y B(a, \ldots, f)$ is not an identical equation, only classes of size 2 and 4 can occur:

$$
a b c d \mid e f \text { or } a b|c d| e f
$$

(up to permutation of the parameters), called $4 / 2$ or $2|2| 2$ partitions, respectively.

In the following, we let permutations $\sigma \in S_{(a, \ldots, f)}$ act on $\operatorname{LHS}(a, \ldots, f)$ and $R H S(a, \ldots, f)$, and hence on $Y B(a, \ldots, f)$, in the obvious sense.

Lemma 4. Let $\left(\rho_{c d}^{a b}\right)$ be a solution of the YBE obeying conditions (5) and (7). Let $G \subset S_{\{a, \ldots, f\}}$ be the group generated by $\tau_{1}=(a c)(d f)$ and $\tau_{2}=(a f)(b e)(c d)$. Then each $\sigma \in G$ leaves $Y B(a, \ldots, f)$ invariant.

Proof. Applying (5) and (7) to the RHS of the YBEs, modifying the names of the summation indices appropriately, we get

$$
R H S(a, \ldots, f)=\rho_{j k}^{a b} \rho_{i f}^{k c} \rho_{d e}^{j i}=\rho_{i j}^{b a} \rho_{f k}^{c i} \rho_{e d}^{k j},
$$

whence

$$
\tau_{1} \operatorname{LHS}(a, \ldots, f)=\operatorname{RHS}(a, \ldots, f) .
$$

Similarly, we have

$$
R H S(a, \ldots, f)=\rho_{i j}^{e d} \rho_{c k}^{f i} \rho_{b a}^{k j}
$$

implying

$$
\tau_{2} \operatorname{LHS}(a, \ldots, f)=\operatorname{RHS}(a, \ldots, f) .
$$


Remark. Clearly, $G$ is the Klein 4-group:

$$
G=\left\{\mathrm{id}, \tau_{1}, \tau_{2}, \tau_{1} \tau_{2}\right\},
$$

where $\tau_{1} \tau_{2}=(a d)(b e)(c f)$ leaves $Y B(a, \ldots, f)$ "side-wise" invariant.

From now on we assume that $\left(\rho_{c d}^{a b}\right)$ satisfies (5), (7), and (15). Taking the previous lemmas into account it is natural to consider the orbits of $4 / 2$ and $2|2| 2$ partitions under the action of $G$.

- Orbits under $G$ of the 15 partitions of $\{a, \ldots, f\}$ of type $4 \mid 2$ :

$$
\begin{aligned}
& a b c d|e f \sim a b c f| d e \sim c d e f|a b \sim a d e f| b c, \\
& a b c e|d f \sim b d e f| a c((), \\
& a b d e|c f \sim b c e f| a d, \\
& a b d f|c e \sim b c d f| a e \sim a c e f|b f \sim a c d e| b f, \\
& \text { abef }|c d \sim b c d e| a f((), \\
& \text { acdf } \mid b e \quad(\odot) .
\end{aligned}
$$

- Orbits under $G$ of the 15 partitions of $\{a, \ldots, f\}$ of type $2|2| 2$ :

$$
\begin{aligned}
& a b|c d| e f \sim a f|b c| d e \quad(\odot) \text {, } \\
& a b|c e| d f \sim a e|b c| d f \sim a c|b d| e f \sim a c|b f| d e, \\
& a b|c f| d e \sim a d|b c| e f, \\
& \text { ac|be|df (O), } \\
& \text { ad|be|cf (O), } \\
& a d|b f| c e \sim a e|b d| c f \text {, } \\
& a e|b f| c d \sim a f|b d| c e \quad((), \\
& \text { af } \mid \text { be } \mid c d \text { (○). }
\end{aligned}
$$

Observe that we have marked by 0 exactly those partitions which are left fixed by $\tau_{1}$ or $\tau_{2}$. Consequently, they correspond to identical equations. We conclude in

Theorem 5. If the matrix $\left(\rho_{c d}^{a b}\right)$ is restricted to equations (5), (7), and (15), the system of $Y B E s(9)$ reduces to equations $Y B(a, \ldots, f)$ corresponding to the following partitions:

$$
\begin{array}{ccc}
a b c d \mid e f & a b d e \mid c f & a b d f \mid c e, \\
a b|c e| d f & a b|c f| d e & a d|b f| c e .
\end{array}
$$

\section{New Invariants}

Following Theorem 5 we can design topological Yang-Baxter invariants by:

- Fixing a dimension $\boldsymbol{n}$. 
- Introducing $3\left(\begin{array}{l}n \\ 2\end{array}\right)+n$ variables $(1 \leq p \neq q \leq n)$ :

$$
\begin{aligned}
& A_{p q}=\rho_{p q}^{p q}=\bar{\rho}_{q q}^{p p}, \\
& B_{p q}=\rho_{q q}^{p p}=\bar{\rho}_{p q}^{p q}, \\
& C_{p q}=\rho_{p q}^{p q}=\bar{\rho}_{p q}^{p q}, \\
& D_{p}=\rho_{p p}^{p p}=\bar{\rho}_{p p}^{p p} .
\end{aligned}
$$

- Solving the YBEs for $\left(\rho_{c d}^{a b}\right)$, which comes down to (Theorem 5):

$$
\begin{aligned}
\sum_{i=1}^{n} A_{p i} A_{q i} B_{p i} & =A_{p q}\left(B_{p q} D_{p}+C_{p q}^{2}\right) & & (p \neq q), \\
C_{p q}\left(B_{p q}^{2}-A_{p q}^{2}\right) & =0 & & (p \neq q), \\
\sum_{i=1}^{n} A_{p i}^{2} B_{q i} & =B_{p q}\left(B_{p q} D_{p}+C_{p}^{2}\right) & & (p \neq q), \\
\left(A_{p q} A_{q r}+A_{p r} B_{q r}\right) C_{q r} & =A_{p r} B_{p q} C_{q r} & & (p \neq q \neq r \neq p), \\
\sum_{i=1}^{n} A_{p i} A_{r i} B_{q i} & =A_{p r} B_{p q} B_{q r} & & (p \neq q \neq r \neq p), \\
\left(B_{p q} B_{q r}+B_{p r} B_{q r}\right) C_{q r} & =B_{p r} B_{p q} C_{q r} & & (p \neq q \neq r \neq p),
\end{aligned}
$$

where we put

$$
A_{p p}=B_{p p}=C_{p p}=D_{p}, \quad 1 \leq p \leq n .
$$

- Adding the YBEs for $\left(\bar{\rho}_{c d}^{a b}\right)$, which comes down to the previous equations with $A$ and $B$ switched, giving (TYB1')-(TYB6 ${ }^{\prime}$ ) (notice that (TYB2) $\equiv\left(\mathrm{TYB2}^{\prime}\right)$ ).

- Constructing finally $T(\mathcal{D})$ by means of $\rho$ and $\bar{\rho}$.

Usually, we insist that $\rho \neq \bar{\rho}$ in order to avoid trivial invariants.

Example. If we take

$$
\begin{array}{ll}
A_{p q}=A, & B_{p q}=B, \\
C_{p q}=0, & D_{p}=A+B
\end{array}
$$

$(p \neq q)$, we obtain:

- $($ TYB1) $\equiv($ TYB3) $\equiv($ TYB5):

$$
B\left(A^{2}+n A B+B^{2}\right)=0 .
$$

- $\left(\mathrm{TYB1}^{\prime}\right) \equiv\left(\mathrm{TYB}^{\prime}\right) \equiv\left(\mathrm{TYB5}^{\prime}\right)$ :

$$
A\left(A^{2}+n A B+B^{2}\right)=0 .
$$

- The sides of the remaining equations are identically zero. 
So, if $A$ and $B$ are such that

$$
A^{2}+n A B+B^{2}=0
$$

we get a solution, giving back the specialization of Kauffman's polynomial as described in Section 4.

Example. If we take

$$
B_{p q}=C_{p q}=D_{p}=0,
$$

for all $1 \leq p, q \leq n$, we get the so-called all $A$-state $\Sigma_{A}$, a configuration of projectively closed curves that is obtained by resolving each crossing by the $A$-split. Furthermore, the only labelings of $\Sigma_{A}$ with nonzero vertex weights have nonequal spin values at each resolution, called polarized labelings. Since the weight of an $A$-split vertex with spin values $p \neq q$ equals $A_{p q}$, the global valuation of such a polarized labeling $L$ looks like

$$
w(L)=\prod_{\text {vertices }} A_{p q}
$$

For example, we consider the $\Sigma_{A}$ for the planar layout $\mathcal{D}$ in Fig. 12. In the case $n=2$ we obviously do not have polarized labelings, yielding that $T(\mathcal{D})=0$. On the other hand, if $n=3$, polarized labelings indeed exist, one of which is given in Fig. 12. The corresponding valuation is

$$
w(L)=A_{12}^{3} A_{13}^{4} A_{23}^{3}
$$

It is not hard to see that this polarized labeling is unique up to permutations of the spin values. So, for the case $n=3$ we get

$$
T(\mathcal{D})=\sum_{\sigma \in S_{3}} A_{\sigma(1) \sigma(2)}^{3} A_{\sigma(1) \sigma(3)}^{4} A_{\sigma(2) \sigma(3)}^{3}
$$

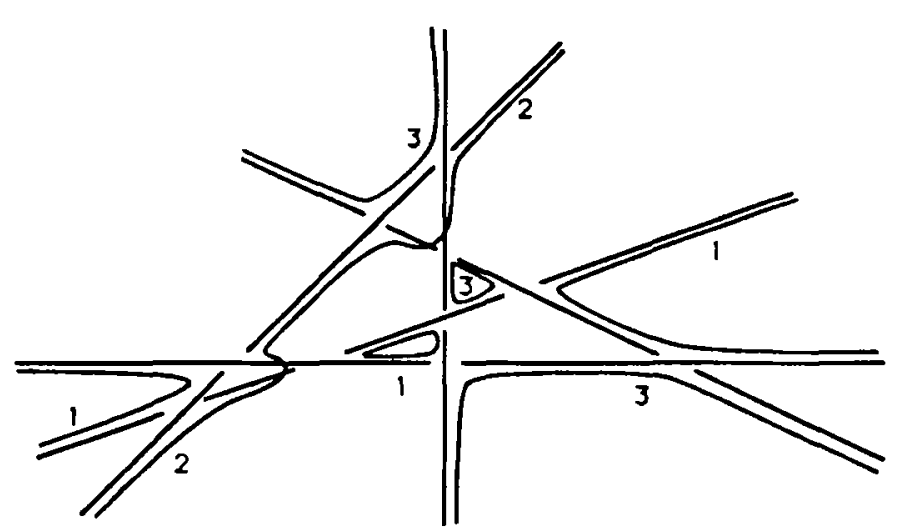

Fig. 12. The state $\Sigma_{A}$ for a line diagram. with a polarized labeling for $n=3$. 
We conclude in

Theorem 6. Let $\mathcal{P}$ be the set of all polarized labelings of the all $A$-state $\Sigma_{A}$ (resp. the all $B$-state $\Sigma_{B}$ ). Then

$$
T(\mathcal{D})=\sum_{L \in \mathcal{P}} w(L)
$$

is an isotopy invariant for $\mathcal{D}$.

Theorem 6 suggests another topological state model which is not known to a YangBaxter invariant. We call a split state $\Sigma$ of a diagram $\mathcal{D}$ hamiltonian if no (projective) closed curve of $\Sigma$ visits the same vertex twice. We define an integer as follows:

$$
\tau_{A}(\mathcal{D})= \begin{cases}0 & \text { if the all } A \text {-state } \Sigma_{A} \text { is not hamiltonian, } \\ \left|\Sigma_{A}\right| & \text { otherwise. }\end{cases}
$$

Of course, $\tau_{B}$ can be defined analogously. A direct check of the $\|$-moves and $*$-moves gives:

Theorem 7. $\tau_{A}\left(\right.$ or $\left.\tau_{B}\right)$ is an invariant for line isotopy.

Example. The case $n=2$ is easy enough to characterize all topological Yang-Baxter invariants. Indeed, only five variables are involved $A_{12}, B_{12}, C_{12}, D_{1}, D_{2}$, where it is assumed that $A_{12} \neq B_{12}$. Clearly, equations (TYB4), (TYB5), (TYB6) and (TYB4'), $\left(\mathrm{TYB5}^{\prime}\right)$, (TYB6 $\left.{ }^{\prime}\right)$ do not occur. Further, each of (TYB1)-(TYB3 ${ }^{\prime}$ ) represents two equations, one for each choice of $(p, q) \in\{(1,2),(2,1)\}$. If we subtract the equations (TYB 1') from the corresponding equations (TYB1), we get

$$
\begin{aligned}
& \left(A_{12}-B_{12}\right)\left(D_{1}^{2}-C_{12}^{2}\right)=0, \\
& \left(A_{12}-B_{12}\right)\left(D_{2}^{2}-C_{12}^{2}\right)=0 .
\end{aligned}
$$

This reduces the subsystem (TYB1), (TYB2), (TYB1'), (TYB2') to

$$
\begin{aligned}
D_{1}^{2} & =C_{12}^{2}, \\
D_{2}^{2} & =C_{12}^{2}, \\
A_{12} B_{12}\left(D_{2}-D_{1}\right) & =0, \\
C_{12}\left(A_{12}^{2}-B_{12}^{2}\right) & =0 .
\end{aligned}
$$

- The case $C_{12} \neq 0$ :

$$
\begin{gathered}
\Rightarrow \quad A_{12}^{2}=B_{12}^{2} \\
\Rightarrow \quad A_{12}=-B_{12}, D_{1}=D_{2}, C_{12}= \pm D_{1},
\end{gathered}
$$

turning (TYB3) and (TYB3') into redundant identical equations. 


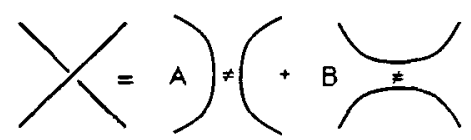

Fig. 13. The second Yang-Baxter invariant for $n=2$, expressed as a topological state model.

- The case $C_{12}=0$

$$
\Rightarrow \quad D_{1}=D_{2}=0
$$

turning (TYB2), (TYB3), and (TYB3') into identical equations.

Theorem 8. The two-dimensional Yang-Baxter invariants for line isotopy where $\rho \neq$ $\bar{\rho}$ satisfy the "topological" restriction (15) are exactly given by

$$
\left(\begin{array}{cccc}
p & 0 & 0 & -q \\
0 & q & \pm p & 0 \\
0 & \pm p & q & 0 \\
-q & 0 & 0 & p
\end{array}\right) \quad \text { or } \quad\left(\begin{array}{cccc}
0 & 0 & 0 & B \\
0 & A & 0 & 0 \\
0 & 0 & A & 0 \\
B & 0 & 0 & 0
\end{array}\right)
$$

where $p, q, A$, and $B$ are parameters $(q \neq 0$ for the former, $A \neq B$ for the latter $)$.

We try to interpret the second solution of Theorem 8 by means of a topological model. The expansion of a crossing is now given by Fig. 13. Obviously, the associated invariant $T(\mathcal{D})$ can be computed by considering only the "polarized split states" (admitting a

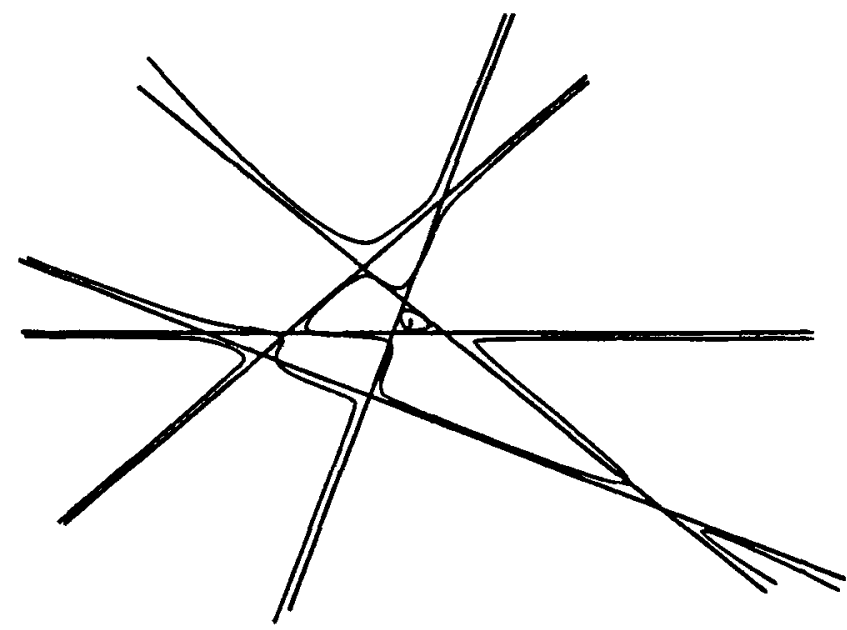

Fig. 14. The onion state with respect to the pointed component $c$. 


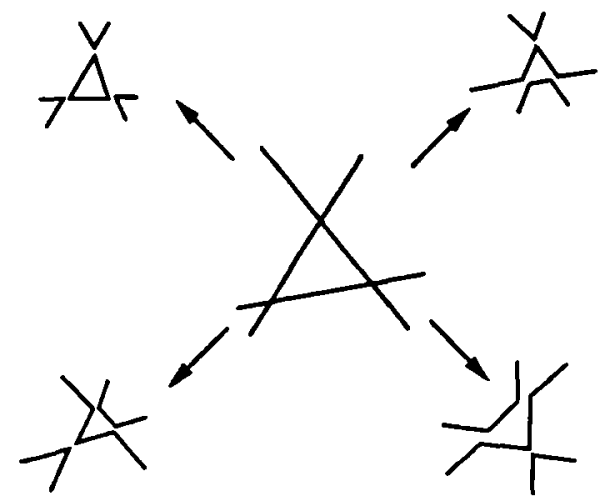

Fig. 15. The four partial split states of a triangle that can be part of an onion state.

polarized labeling). More precisely, if we put

$$
[\mathcal{D}]_{p}=\sum_{\text {polarized } \Sigma} A^{a(\Sigma)} B^{b(\Sigma)}
$$

then $T(\mathcal{D})=2[\mathcal{D}]_{p}$. It is preferable to use the normalized $\left[\mathcal{D}_{p}\right]$. It is an interesting exercise on diagram moves to give a direct proof that $[\mathcal{D}]_{p}$ is an invariant for line isotopy.

We close this section by presenting another invariant given by a topological state model, which is related to the previous constructions in the sense that we consider subclasses of the total set of split states.

Let $c$ be a cell of the arrangement $\mathcal{H}$ of (pseudo)lines underlying a diagram $\mathcal{H}$, that is, $c$ is a connected component of $\mathbf{R P}^{2} \backslash \cup \mathcal{H}$. Further, let $\left\{\lambda_{1}, \lambda_{2}\right\}$ be a pair of (pseudo)lines of $\mathcal{H}$. The complement $\mathbf{R P}^{2} \backslash\left(\lambda_{1} \cup \lambda_{2}\right)$ consists of two connected components $A$ and $B$, corresponding to the two possible vertex splits (Fig. 10). The split state " $\Sigma(c)$ " associated with $c$ is defined by choosing in each vertex $\lambda_{1} \cap \lambda_{2}$ the split that corresponds to the unique component $(A$ or $B$ ) that does not contain $c$. We call $\Sigma(c)$ the onion state with respect to $C$, and $\mathcal{O}$ denotes the set of all onion states. See Fig. 14 for an example of an onion state. The onion of a diagram $\mathcal{D}$ is defined as

$$
\omega(\mathcal{D})=\sum_{\Sigma \in \mathcal{O}} A^{a(\Sigma)} B^{b(\Sigma)} .
$$

Theorem 9. $\omega(\mathcal{D})$ is an invariant for the line isotopy.

Proof. Invariance under \|-moves is immediate, since $\omega(\mathcal{D})$ has a projective definition. To see the invariance under $*$-moves it should be observed that only four of the eight vertex splits of a triangle can be part of an onion state (Fig. 15). For these partial split states the type of split $(A$ or $B$ ) does not change under $*$-moves. 


\section{References}

1. Y. Akutsu and M. Wadati, Knots, links, braids and exactly solvable models in statistical mechanics. Comm. Math. Phys., 117:243-259, 1988.

2. A. Borobia and V. Mazurovskii. On diagrams of configurations of 7 skew lines of $\mathbf{R}^{3}$. Adv. in Soviet Math., to appear.

3. H. Crapo and R. Penne. Chirality and the isotopy classification of skew lines in projective 3-space. Adv, in Math., 103(1):1-106, 1994.

4. Yu. V. Drobotukhina. An analogue of the Jones polynomial for links in $\mathbf{R P}^{3}$ and a generalization of the Kauffman-Murasugi theorem. Leningrad Math. J., 2(3):613-630, 1991.

5. M. Jimbo. Introduction to the Yang-Baxter equation. Internat. J. Mod. Phys. A, 4(15):3759-3777, 1989.

6. L. H. Kauffman. On Knots. Annals of Mathematics Studies, vol. 115. Princeton University Press, Princeton, NJ, 1987.

7. L. H. Kauffman. State models and the Jones polynomial. Topology, 26(3):395 407, 1987.

8. L. H. Kauffman. Knots and Physics. World Scientific, Singapore, 1991.

9. S. Majid. Quasitriangular Hopf algebras and Yang-Baxter equations. Internat. J. Mod. Phys. A, 5(1):1-91, 1990.

10. V.F. Mazurovskii. Kauffman polynomials for nonsingular configurations of projective lines. Russian Math. Surveys, 44(5):212-213, 1989.

11. V. F. Mazurovskii. Configurations of six skew lines. J. Soviet Math., 52(1):2825-2832, 1990.

12. R. Penne. Configurations of few lines in 3-space. Isotopy, chirality, and planar layouts. Geom. Dedicata, 45:49-82, 1993.

13. R. Penne. Moves on pseudoline diagrams. European J. Combin., to appear.

14. V. G. Turaev. The Yang-Baxter equation and invariants of links. Invent. Math., 92:527-553, 1988.

15. O. Ya. Viro. Topological problems concerning lines and points of three-dimensional space. Soviet Math. Dokl., 2(32):528-531, 1985.

Received April 11, 1994, and in revised form February 14, 1995. 